\title{
Effects of Occupational Noise Exposure on Blood Pressure
}

\section{Ta-Yuan Chang, MS \\ Ruei-Man Jain, MS \\ Chiu-Sen Wang, PhD Chang-Chuan Chan, ScD}

We measured 24-hour ambulatory blood pressure and 16-hour noise exposure continuously for 20 automobile workers, and used linear mixed-effects regression models to estimate transient and sustained effects of noise exposure on blood pressure. The occupational noise levels of the high-exposure workers with $85 \pm 8 d B A$ were significantly higher than those of the low-exposure workers with $59 \pm 4 d B A(P<0.05)$. We found a significant difference of $16 \pm 6 \mathrm{~mm} \mathrm{Hg}$ in sleep-time systolic blood pressure (SBP) existed between 2 exposure groups, and a marginal increase of $1 \mathrm{~mm} \mathrm{Hg} S B P$ per 1-dBA increase in occupational noise exposure at a 60-minute lag time during work $(P=0.07)$. Occupational noise exposure had both transient and sustained effects on workers' SBP. (J Occup Environ Med. 2003;45:1289-1296)

From the Institute of Occupational Medicine and Industrial Hygiene, College of Public Health, National Taiwan University, Taipei, Taiwan.

Address correspondence to: Chang-Chuan Chan, ScD, Institute of Occupational Medicine and Industrial Hygiene, College of Public Health, National Taiwan University, Taipei, Taiwan, Room 1447, No.1, 1st Sec., Jen-Ai Rd., Taipei, Taiwan 100; E-mail: ccchan@ha.mc.ntu.edu.tw.

Copyright $($ ) by American College of Occupational and Environmental Medicine

DOI: 10.1097/01.jom.0000100003.59731.3d everal comprehensive reviews have documented various adverse effects of noise exposure, including hearing impairment, annoyance, sleep disturbance, and hypertension. ${ }^{1-3}$ Blood pressure changes were mostly related to occupational noise exposure. Several epidemiologic studies had shown chronic noise exposure significantly increased blood pressure. ${ }^{4-10}$ Two experimental studies conducted in the laboratories had found workers' blood pressure increased after exposure to industrial noise levels greater than $95 \mathrm{dBA} .{ }^{11,12}$ Two field studies, which were originally designed to investigate acute and chronic effects of noise exposure on blood pressure simultaneously, found that occupational noise exposure had transient but no sustained effects on blood pressure. ${ }^{13,14}$ However, 1 animal study reported that noise exposure induced both acute and chronic effects on the rhesus monkey's blood pressure. ${ }^{15}$

In previous epidemiologic studies, only 1-time resting blood pressure measurements were used to represent individuals' blood pressure. Usually, environmental monitoring rather than personal data represented individuals' noise exposure. We believed insufficient measurements for noise exposure, blood pressure, and confounding factors usually restricted the findings of sustained effect by noise exposure in past epidemiologic studies. The lack of continuous measurements also limited the possibility of finding the effect of short-term noise exposure on blood pressure.

In this study, we conducted a panel study on automobile workers to in- 
vestigate whether workers' blood pressure can be increased by their transient and sustained noise exposure in a working environment with high noise exposure.

\section{Methods}

\section{Subjects}

We performed a panel study on 20 male workers in an automobile manufacturing company in Taiwan in 2001. Our study subjects were randomly selected from all male employees who were working either in the production lines or in the office of the automobile company. Major operations in this company included forging and casting, press forging of stainless steel, engine manufacturing and assembly, body assembly, painting, peripheral element assembly, and roller and track trial testing. The 15 subjects with high noise exposure were from operational units of press forging, engine manufacturing and assembly, body assembly, roller and track trial testing. The environmental monitoring data in 2000 showed the noise levels were at 79 to $110 \mathrm{dBA}$ and ototoxic chemicals were not present in these working environments. These study subjects were not wearing any hearing protection devices, but 6 of them were exposed to noise and vibration at the same time during the study. Another 5 subjects with low noise exposure were recruited from the staff working in a separate office building of the company.

According to the health checkup results in 2000 before the panel study, none of these 20 subjects were diagnosed with hypertension or other cardiovascular diseases. The checkup also provided important information related to hypertension such as subjects' heights and weights, resting blood pressure, total cholesterol, and triglyceride. Individuals' heights and weights were used to calculate body mass index (BMI). For the subjects working in the worksites with environmental noise levels above $85 \mathrm{dBA}$, audiometric tests were performed at 500, 1000, 2000, 3000, 4000, 5000, and 6000 $\mathrm{Hz}$ for both ears annually. During the panel study, we used a questionnaire to collect additional risk factors of blood pressure such as individuals' age, employment duration, exercise habit, smoking history, alcohol consumption, and family disease history. The Institutional Review Board of the College of Public Health, National Taiwan University, approved this study, and written informed consent was obtained from each participating worker.

\section{Ambulatory Blood Pressure Monitoring and Recording}

We recorded each subject's blood pressure every 30 minutes during awake time (8:00 AM-11:00 PM) and every 60 minutes during sleep time (11:00 PM-8:00 AM) continuously by using a portable, noninvasive, automated blood pressure monitoring and recording system (DynaPulse model 5000A, Pulse Metric, San Diego, CA). The DynaPulse system can measure a subject's arterial pulsation signals, known as the arterial waveform, through a noninvasive cuff device. The systolic and diastolic blood pressure readings by DynaPulse have been validated against the traditional mercury sphygmomanometer measurements. ${ }^{16,17}$ Each subject carried a DynaPulse system for 24 hours to complete his continuous blood pressure monitoring. The blood pressure data of 15 high noise exposure subjects during the working period (8:00 AM-4:30 PM) were used to investigate the transient effect of occupational noise exposure on workers' systolic blood pressure (SBP) and diastolic blood pressure (DBP). All 20 participants' blood pressure measurements during the sleep period were used to study the sustained effect by occupational noise exposure.

\section{Exposure Measurements}

We measured personal noise exposure continuously by using a personal noise dosimeter (Logging Noise Dose Meter type 4443, Brüel
\& Kjær, Nærum, Denmark), which can report 5-minute continuous equivalent sound level (Leq) at an exchange rate of $3 \mathrm{dBA}$ and timeweighted-average (TWA) noise dose. This dosimeter has 3 measurement ranges, which are $70-140$ dBA, 50-120 dBA, and 30-100 dBA. The range of $50-120 \mathrm{dBA}$ was used to measure the high-exposure group and the range of 30-100 dBA was to measure the low-exposure group. All subjects carried the Logging Noise Dose Meter to measure their personal noise exposure with 5-minute readings over their working period (8:00 AM-4:30 PM). One single TWA noise exposure level was obtained for each subject during the off-duty period (4:30 PM-11:00 PM). Noise exposure levels were not measured during the sleep period. To investigate the time-lag effect of acute noise exposure, 5-minute exposure measurements were transformed to 30-minute, 60-minute, and 120minute time-moving-average segments for further analysis.

\section{Statistical Analysis}

We performed univariate comparisons between the 2 exposure groups by using $t$ test for continuous variables and Fisher exact test for dichotomous variables. We used linear mixed-effects regression models to associate transient noise exposure with blood pressure increase by controlling important confounding factors of study subjects. ${ }^{18}$ The linear mixed-effects regression model has the advantages of dealing with the autocorrelation problem between consecutive blood pressure measurements and increasing statistical power by combining information across study subjects. In our mixedeffects models, personal noise exposure at the moving averages of 0 to 2 hours was generated from original 5-minute measurements. The fixed effect in our mixed-effects models contained covariance parameters of age, employment duration, BMI, smoking, alcohol consumption, fam- 
TABLE 1

Brief Description of Subjects

\begin{tabular}{|c|c|c|c|}
\hline Characteristics & $\begin{array}{l}\text { High-Exposed } \\
\text { Group }\end{array}$ & $\begin{array}{c}\text { Low-Exposed } \\
\text { Group }\end{array}$ & $\begin{array}{c}P \\
\text { Value }\end{array}$ \\
\hline Subjects (no.) & 15 & 5 & \\
\hline \multicolumn{4}{|l|}{ Age (years) $^{*}$} \\
\hline Mean (SD) & $39(7)$ & $45(8)$ & 0.16 \\
\hline \multicolumn{4}{|c|}{ Employment duration (years) $^{\star}$} \\
\hline Mean (SD) & $14(8)$ & $17(9)$ & 0.58 \\
\hline \multicolumn{4}{|l|}{ Body mass index $\left(\mathrm{kg} / \mathrm{m}^{2}\right)^{*}$} \\
\hline Mean (SD) & $24(2)$ & $26(1)$ & 0.22 \\
\hline \multicolumn{4}{|l|}{ Resting SBP $(\mathrm{mm} \mathrm{Hg})^{*}$} \\
\hline Mean (SD) & $123(19)$ & $121(18)$ & 0.85 \\
\hline \multicolumn{4}{|l|}{ Resting DBP $\left(\mathrm{mm} \mathrm{Hg}^{*}\right.$} \\
\hline Mean (SD) & $83(12)$ & $84(9)$ & 0.98 \\
\hline \multicolumn{4}{|l|}{ Total cholesterol (mg/dL) } \\
\hline Mean (SD) & $205(89)$ & $191(14)$ & 0.56 \\
\hline \multicolumn{4}{|l|}{ Triglyceride $(\mathrm{mg} / \mathrm{dL})^{*}$} \\
\hline Mean (SD) & $170(94)$ & $138(50)$ & 0.49 \\
\hline \multicolumn{4}{|l|}{ Regular exercise $\dagger$} \\
\hline Yes (\%) & $3(20 \%)$ & $2(40 \%)$ & 0.56 \\
\hline No (\%) & $12(80 \%)$ & $3(60 \%)$ & \\
\hline \multicolumn{4}{|l|}{ Smokingt } \\
\hline Yes (\%) & $11(73 \%)$ & $2(40 \%)$ & 0.29 \\
\hline No $(\%)$ & $4(27 \%)$ & $3(60 \%)$ & \\
\hline \multicolumn{4}{|l|}{ Alcohol consumption $\dagger$} \\
\hline Yes (\%) & 5 (33\%) & $1(20 \%)$ & 1.00 \\
\hline No $(\%)$ & $10(67 \%)$ & $4(80 \%)$ & \\
\hline \multicolumn{4}{|l|}{ Hypertension family } \\
\hline Yes (\%) & $8(57 \%)$ & $3(60 \%)$ & 1.00 \\
\hline No $(\%)$ & $6(43 \%)$ & $2(40 \%)$ & \\
\hline \multicolumn{4}{|c|}{ Material hearing impairmentł } \\
\hline Number (\%) & 12 (80\%) & NA & \\
\hline
\end{tabular}

${ }^{*} t$ test of the difference between the 2 exposure groups.

† Fisher exact test of the difference between the 2 exposure groups.

‡ Defined by the U.S. Occupational Safety and Health Administration's criteria, ie, an average hearing threshold level (HTL) of 25-dB HTL at 1000, 2000, and $3000 \mathrm{~Hz}$.

SD, standard deviation; SBP, systolic blood pressure; DBP, diastolic blood pressure.

$\mathrm{NA}=$ Not available.

ily hypertension history, and noise exposure. Individual subjects were treated as a random effect in the model. The explanatory variables were chosen in a stepwise procedure to minimize Akaike's information criterion (AIC), which is a function of the log-likelihood function adjusted for the number of covariance parameters. ${ }^{19}$ The model with the lowest AIC value was selected as the best-fitted model. The mixed procedure containing fixed and random effects in SAS 8.1 version (SAS Institute Inc., Cary, NC) was used to perform the linear mixed-effects regression, and the statistical significance level was set at 0.05 in this study.

\section{Results}

Table 1 summarizes the demographic characteristics and health risk factors by noise exposure levels for 20 study subjects. There were no significant differences between high and low noise exposure workers for age, employment duration, BMI, resting SBP, resting DBP, total cholesterol, triglyceride, regular exercise, smoking, habitual drinking, and a family history of hypertension $(P>0.05)$. Although there is no statistically significant betweengroup difference in the prevalence of smoking, it is worth pointing out that the prevalence of smoking is very high in the high-exposure group
(73\%) compared with the lowexposure group (40\%). There were 12 workers in the high-exposure group meeting the criteria of material hearing impairment defined by the U.S. Occupational Safety and Health Administration, which is an average hearing threshold level (HTL) of 25-dB HTL at 1000, 2000, and 3000 Hz. Their average hearing thresholds were $50-\mathrm{dB}$ HTL at $4000 \mathrm{~Hz}$ and 51-dB HTL at $6000 \mathrm{~Hz}$, respectively.

An exemplary graph of concurrent measurements of noise exposure and SBP for 1 high-exposure worker and 1 low-exposure worker is shown in Figure 1. The 24-hour SBP profiles of the high-exposure worker were consistently higher than those of the low-exposure worker. For both workers, the variation in SBP was more profound during the working period (8:00 AM-4:30 PM) than either the off-duty period (4:30 PM-11:00 $\mathrm{PM})$ or the sleep period (11:00 PM8:00 AM).

During the study subjects' 24-hour monitoring periods, we obtained a total of 477 available blood pressure measurements and 1785 personal noise readings, including $1765 \mathrm{seg}$ ments of 5-minute Leq during the working period and 20 TWA data during the off-duty period. Data completeness was $87 \%$ (1785 of 2060) for noise exposure measurements and 61\% (477 of 780) for blood pressure measurements. The missing data averaged at 14 (range, 6-24) for noise measurements and 16 (range, 7-24) for blood pressure measurements. Table 2 summarizes SBP, DBP, and personal noise exposure measured over 24 hours, the 8.5-hour working period, the 6.5hour off-duty period, and the 9-hour sleep period for workers with high and low noise exposure during the study. As expected, the full-shift TWAs (Leq) of high-exposure workers $(85 \pm 8 \mathrm{dBA}$; mean \pm standard deviation) were significantly higher than those of low-exposure workers during the working period $(59 \pm 4$ $\mathrm{dBA}$ ). The 15 high-exposure workers' full-shift TWAs (Leq) were also 


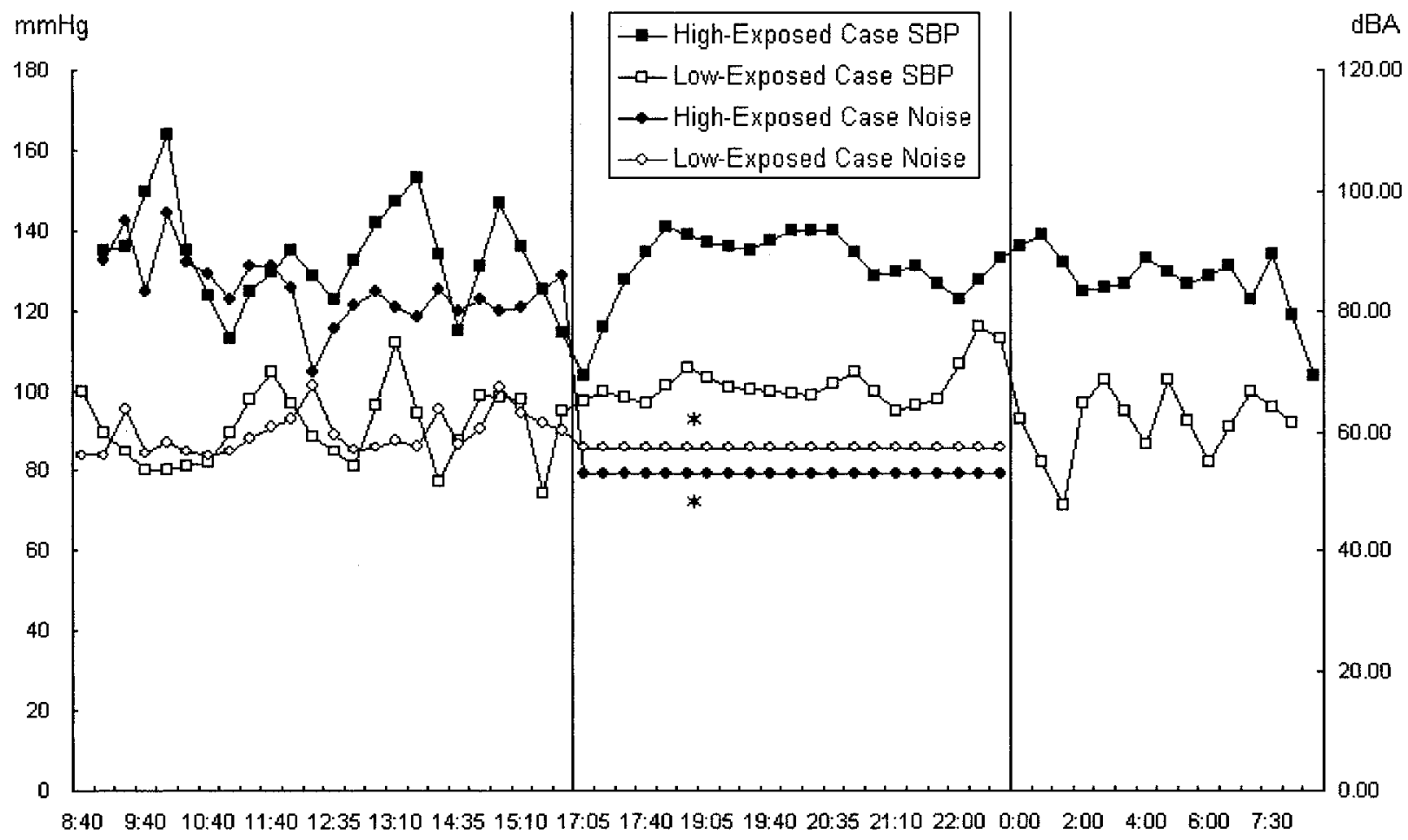

Fig. 1. Measurements of noise exposure and systolic blood pressure in the 2 different cases. * One single time-weighted-average noise level obtained for each subject during the off-duty period (4:30 PM-11:00 PM).

comparable to their environmental noise levels measured in October 2000 (79-110 dBA). By contrast, the full-shift TWAs (Leq) during the offduty period had no significant difference between the high- and low- exposure groups $(61 \pm 7 \mathrm{dBA}$ vs. $56 \pm 12 \mathrm{dBA})$.

The distribution of the current exposure showed that the full-shift TWAs (Leq) were all above $70 \mathrm{dBA}$ for the 15 high-exposure workers at work during the study. Moreover, there were approximately $90 \%$ measurements above $75 \mathrm{dBA}, 49 \%$ measurements above $85 \mathrm{dBA}$, and $9 \%$ measurements above $95 \mathrm{dBA}$. By contrast, approximately $99 \%$ of the

\section{TABLE 2}

Mean Values \pm Standard Deviations of SBP, DBP, and Personal Noise Exposure Over 24-Hour, Working Time, Off-Duty Time and Sleep Time in the High- and Low-Exposure Groups

\begin{tabular}{|c|c|c|c|c|}
\hline & $\begin{array}{c}\text { Working Time } \\
\text { (8:00 AM-4:30 PM) }\end{array}$ & $\begin{array}{c}\text { Off-Duty Time } \\
\text { (4:30 PM-11:00 PM) }\end{array}$ & $\begin{array}{c}\text { Sleep Time } \\
\text { (11:00 PM-8:00 AM) }\end{array}$ & 24-Hour Average \\
\hline \multicolumn{5}{|l|}{ SBP $(m m ~ H g)$} \\
\hline High-exposure group (no.)* & $127 \pm 24(125)$ & $122 \pm 16(112)$ & $110 \pm 16(121)$ & $120 \pm 21(358)$ \\
\hline Low-exposure group (no.) & $108 \pm 17(52)$ & $114 \pm 15(41)$ & $100 \pm 16(26)$ & $108 \pm 17(119)$ \\
\hline$P$ value $\dagger$ & $<0.01$ & 0.11 & 0.01 & $<0.01$ \\
\hline \multicolumn{5}{|l|}{$\mathrm{DBP}(\mathrm{mm} \mathrm{Hg})$} \\
\hline High-exposure group (no.) & $75 \pm 20(125)$ & $73 \pm 12(112)$ & $64 \pm 11(121)$ & $71 \pm 16(358)$ \\
\hline Low-exposure group (no.) & $68 \pm 13(52)$ & $70 \pm 16(41)$ & $62 \pm 13(26)$ & $67 \pm 14(119)$ \\
\hline$P$ value & 0.05 & 0.05 & 0.21 & 0.02 \\
\hline \multicolumn{5}{|l|}{ Personal noise (dBA) } \\
\hline High-exposure group (no.) & $85 \pm 8(1301)$ & $61 \pm 7(15)$ & NA & NA \\
\hline Low-exposure group (no.) & $59 \pm 4(464)$ & $56 \pm 12(5)$ & NA & NA \\
\hline$P$ value $\neq$ & $<0.01$ & 0.21 & & \\
\hline
\end{tabular}

* Number of measurements for SBP, DBP, and personal noise exposure.

† Linear mixed-effects regression models adjusted for age, employment duration, body mass index, smoking, drinking alcohol, and hypertension family were used to test the between-group differences.

$\ddagger t$ test was used to test the difference between the 2 exposure groups.

SBP, systolic blood pressure; DBP, diastolic blood pressure. 


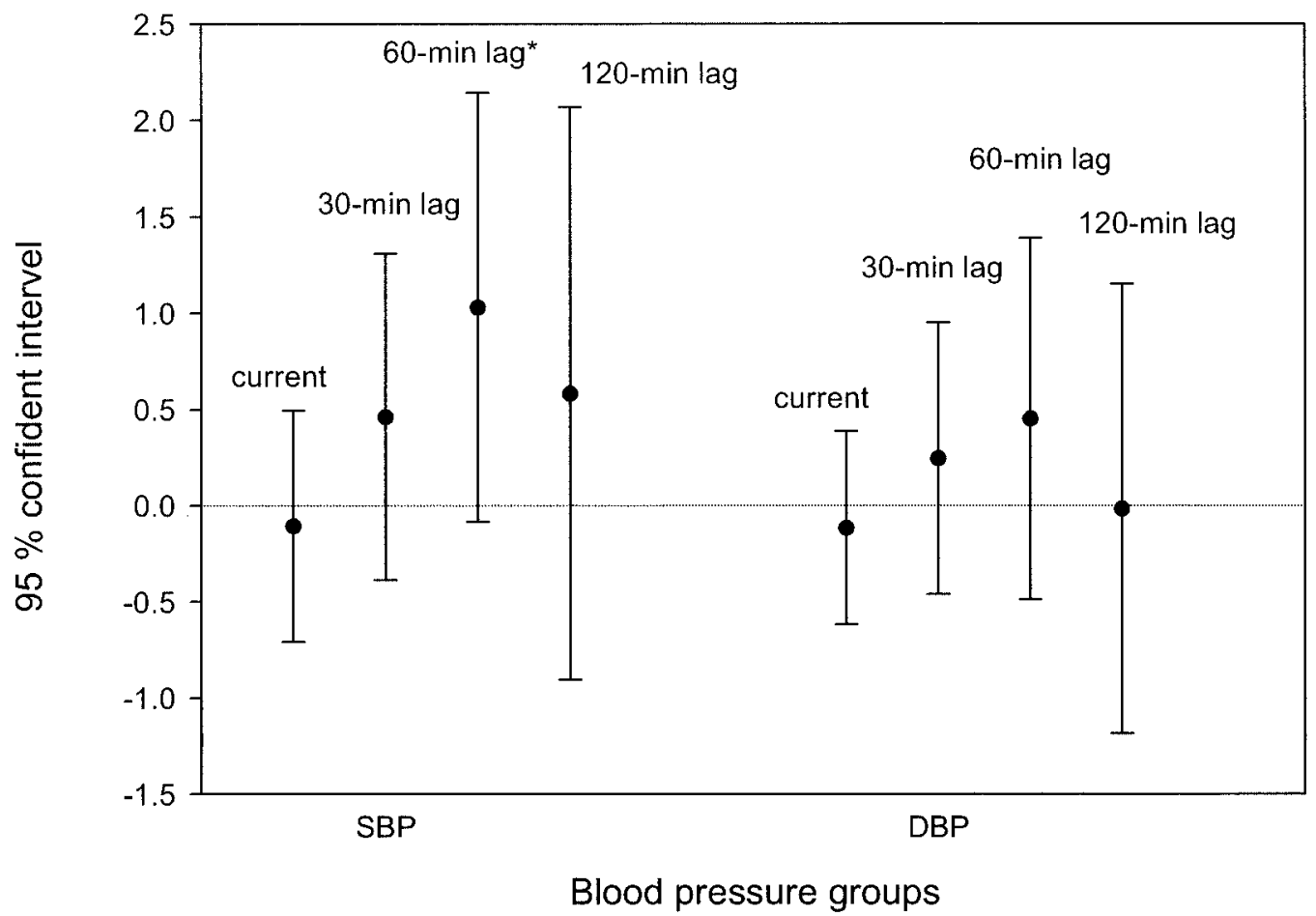

Fig. 2. The transient effect of noise exposure on blood pressure in the high-exposed group by linear mixed-effects models after adjusting for age, employment duration, body mass index, smoking, drinking alcohol, and hypertension in the family. ${ }^{*}$ Marginally significant $(P=0.07)$.

\section{TABLE 3}

The Association Between Occupational Noise Exposure and Sleep-Period Blood Pressure

\begin{tabular}{|c|c|c|c|c|}
\hline \multirow[b]{2}{*}{ Models } & \multicolumn{2}{|c|}{ SBP (mm Hg) } & \multicolumn{2}{|c|}{ DBP (mm Hg) } \\
\hline & $\beta( \pm \operatorname{Se} \beta)$ & $\begin{array}{c}P \\
\text { Value }\end{array}$ & $\beta( \pm \operatorname{Se} \beta)$ & $\begin{array}{c}P \\
\text { Value }\end{array}$ \\
\hline High exposure vs. low exposure & $16( \pm 6)$ & 0.02 & $6( \pm 5)$ & 0.22 \\
\hline Hypertension family (yes/no) & $13( \pm 5)$ & 0.02 & $5( \pm 4)$ & 0.25 \\
\hline Employment duration (years) & $2( \pm 1)$ & 0.01 & $1( \pm 1)$ & 0.12 \\
\hline Body mass index $\left(\mathrm{kg} / \mathrm{m}^{2}\right)$ & $2( \pm 1)$ & 0.12 & $2( \pm 1)$ & 0.06 \\
\hline Alcohol (yes/no) & $1( \pm 5)$ & 0.88 & $4( \pm 4)$ & 0.36 \\
\hline Age (years) & $-1( \pm 1)$ & 0.21 & $0( \pm 1)$ & 0.55 \\
\hline Smoking (yes/no) & $-1( \pm 6)$ & 0.87 & $-1( \pm 5)$ & 0.80 \\
\hline
\end{tabular}

SBP, systolic blood pressure; DBP, diastolic blood pressure.

full-shift TWAs (Leq) were measured between 50 and $70 \mathrm{dBA}$ for the 5 low-exposure workers. These distributions in the full-shift TWAs (Leq) indicated that the high and low noise-exposure groups in our subjects could be classified by an occupational noise exposure at $70 \mathrm{dBA}$. In fact, the full-shift TWAs (Leq) were mostly greater than $75 \mathrm{dBA}$ for the high-exposure group and were all less than $65 \mathrm{dBA}$ for the lowexposure group.
The high-exposure workers' SBP were significantly higher than the low-exposure workers' ones by averaging $12 \mathrm{~mm} \mathrm{Hg}$ over 24 hours, 19 $\mathrm{mm} \mathrm{Hg}$ over the working period, and $9 \mathrm{~mm} \mathrm{Hg}$ over the sleep period. The high-exposure workers' DBP were also significantly higher than the low-exposure workers' ones by averaging $4 \mathrm{~mm} \mathrm{Hg}$ over 24 hours and 7 $\mathrm{mm} \mathrm{Hg}$ over the working-period. Over the off-duty period, the DBP was marginally different $(P=0.05)$, but the SBP was not different $(P=$ $0.11)$ between the high- and lowexposure groups. Over the sleep time, the DBP was not different between the high- and low-exposure groups either.

The concurrent measurements of noise exposure and blood pressure during the working period among the high-exposure workers were used to estimate the transient effect of noise exposure. Figure 2 shows the SBP and DBP changes by various timelagged noise exposure after adjusting for age, employment duration, BMI, smoking, drinking, and family hypertension history in the linear mixed-effects models. The mixedeffects models showed workers' SBP and DBP increased transiently at 30 and 60 minutes and returned to baselines at 120 minutes after noise exposure at work. Our models showed that there was a marginally significant increase of $1 \mathrm{~mm} \mathrm{Hg} \mathrm{SBP}$ at 60-minute lag for the 1-dBA increase in noise exposure for autoworkers with occupational exposure between 70 and $108 \mathrm{dBA}(P=0.07)$. 
The association between occupational noise exposure and sleepperiod blood pressure is summarized in Table 3. Based on a dichotomous noise exposure variable (high vs. low), our regression models showed that occupational noise exposure was significantly associated with SBP $(P=0.02)$ but not associated with DBP $(P=0.22)$ after controlling other risk factors. Workers with TWA occupational noise exposure of $85 \pm 8 \mathrm{dBA}$ had $16 \pm 6 \mathrm{~mm} \mathrm{Hg}$ increments in sleep-time SBP compared with workers with TWA occupational noise exposure of $59 \pm 4$ dBA. In addition, we also identified that sleep-time SBP were significantly increased for workers with family hypertension history and longer employment duration $(P<$ $0.05)$. Although there was a significant correlation between age and employment duration $(\mathrm{r}=0.883, P<$ 0.01 ), we found that the interaction term of these 2 variables was not significant when it was added to our regression models $(P=0.10)$.

\section{Discussion}

We demonstrated that using recently available personal monitoring instruments to measure individuals' noise exposure and blood pressure continuously could reveal the transient and sustained effects of occupational noise exposure on blood pressure. Our study showed that 24hour continuous measurements of blood pressure were key to reveal the effects of noise on blood pressure. There was a considerable discrepancy between 2 blood pressure data of Table 1 and Table 2. There were explanations for this seeming discrepancy. The values in Table 1 were obtained as single measurements by using a nonsystematic protocol in a clinic setting. In contrast, the present study collected repeated measurements over a span of 24 hours on each individual using a systematic protocol and different measurement instruments. Our study showed that the ambulatory monitoring technique was able to identify between-group differences in blood pressure that were not evident with conventional clinical measurements, including identification of subjects with prehypertension (SBP in 120-139 mm $\mathrm{Hg}$ ) for the high-exposure group and normal blood pressure status (below $120 \mathrm{~mm} \mathrm{Hg}$ ) for the low-exposure group, respectively. Accordingly, the long-term effect of noise was better evaluated by using 24-hour blood pressure measurements. In addition, the investigation on acute effect of noise became feasible because continuous blood pressure was measured concurrently with noise exposure in this study.

Our findings suggested the transient effect of increasing SBP could be induced by noise exposure at lower noise levels and shorter time lags. Previous studies found that industrial noise exposure above 85 dBA had a transient effect of increasing SBP. ${ }^{13,14}$ Our study reported such a transient effect could also occur at noise levels as low as 75 dBA. Our study also found the transient effect of noise exposure on SBP had the lagged effect maximally at 1 hour, which was shorter than the 2 or 3 persistent hours reported in a previous study. ${ }^{14}$ However, such transient effect can be overestimated by lacking information about workers' activities during the ambulatory blood pressure monitoring period such as heavy dynamic exercise during working hours and during offduty time.

Increasing sleep-time SBP by TWA occupational noise exposure at $85 \pm 8 \mathrm{dBA}$ indicated that noise exposure could also have a sustained effect on blood pressure because noise exposure differed significantly by $26 \mathrm{dBA}$ during working time but was not significantly different during the off-duty period between the highexposure and the low-exposure groups. By contrast, Babisch et al. reported inconsistent findings of chronic effect for people with lower noise exposure levels, $70 \mathrm{dBA}$, and smaller difference in noise exposure levels between high- and low- exposed groups, $15 \mathrm{dBA} .^{20,21}$ Such comparisons indicate there could be threshold exposure levels for noiseinduced hypertension.

However, we might overestimate the sustained effect of noise exposure on SBP without excluding all potential confounders completely in this study. We included some but not all important risk factors of hypertension that might lead to confounding the sustained effect of noise exposure on blood pressure. Uncontrolled important risk factors of hypertension included serum cholesterol (high-density lipoprotein and low-density lipoprotein), blood glucose, uric acid, creatinine, potentially ototoxic drugs, salt in diet, number of cigarettes smoked per day, and daily alcohol intake and coffee consumption among our study subjects. For example, the high-exposure workers were almost twice as likely to be smokers, although the difference in smoking prevalence was not statistically significant. Therefore, the sustained difference in SBP between 2 exposure groups could still possibly be contributed by lifestyle differences.

Our findings also support that people with longer employment durations and with a family hypertension history are associated with abnormal blood pressure reported in previous studies. ${ }^{6-10}$ Although our statistical model has already controlled age, it should be noted that such analysis might not be sufficiently robust with 20 subjects. Therefore, the possibility of confounding effect on hypertension by age could not be completely excluded.

Two possible mechanisms in the genesis of hypertension resulting from noise exposure are through sympatheticotonia-induced endothelial lesion and stress-induced hormone release. For the former 1, acute noise exposure can activate sympathetic reflex immediately, ${ }^{11,22,23}$ then accelerate the development of structural changes in the cardiovascular system, and finally induce a sustained elevation of blood pres- 
sure. ${ }^{4,11,23}$ On the other hand, several studies have shown higher stress hormone levels in relation to chronic noise exposure. ${ }^{24,25} \mathrm{~A}$ stress-related activation of neuroendocrine pathways through high cortisol niveaus can give rise to a metabolic syndrome with hypertension and other cardiovascular effects as end points. Both pathways are reasonable approaches in the genesis of hypertension resulting from noise exposure.

This is a panel study, and possible limitations with a cross-sectional design often are missing in this situation. Weaknesses with a crosssectional design with lack of exposure information and temporality problem with different confounders were some main topics discussed in past reviews. ${ }^{26,27}$ For example, we have greatly minimized temporality problem by measuring personal noise exposure and ambulatory blood pressure continuously instead of using 1 single measurement of noise exposure and resting blood pressure to investigate the transient and sustained effects of noise exposure on SBP and DBP in this study.

Although the number of study subjects was small, the numbers of available measurements of noise exposure and blood pressure were relatively large. There were 477 available blood pressure measurements and 1785 personal noise readings during the 20 study subjects' 24-hour monitoring period. Accordingly, a small number of study subjects with a large time series data sample size would not affect the determination of transient effect but might limit the extrapolation of the sustained effect observed among autoworkers in this study to the workers in other industrial settings.

One limitation of this study is the problem of missing data, which existed in blood pressure measurements but not in noise exposure measurements. Because the DynaPulse system is based on measuring a subject's arterial waveform to report blood pressure, it is sensitive to the subject's arm movements. The heavy dynamic exercises during working hours and frequent changes of body posture during the sleep period are 2 possible reasons of missing data for blood pressure measurements. Differences in these activities between subjects resulted in inconsistent distribution of missing data of blood pressure across different cells in Table 2 . The ratios of available measurements between high- and lowexposure groups were 4.7 (121:26) in the sleep time, $2.7(112: 41)$ in the off-duty time, and $2.4(125: 52)$ in the working time. The comparisons of blood pressure between different exposure groups and working periods might be compromised by the difference in the availability of data between subjects. The mixed-effects models were constructed to analyze data with different observational length data between study subjects. Therefore, difference in the availability of data between subjects would not compromise the validity of our statistical analyses in determining the transient and sustained effects of noise exposure on blood pressure.

The other limitation of this study is the truncated noise exposure measurement between 30 and $50 \mathrm{dBA}$ for the high-exposure group during the off-duty period. This is because it was impractical for us to adjust the dosimeter's ranges of our study subjects after their working periods in our study. Ideally, the high-exposed subjects should have been provided separate dosimeters for our off-duty sampling with the measurement range that was identical to that for the low-exposed subjects. However, there is likely to be little, if any, effect from such measurement bias on the primary data analysis, which focuses on the relationship between blood pressure and work-period noise exposure. It should be noted that our choice of the practical approach could introduce a potential bias if the truncated data during the off-duty period were used for our primary data analysis. Therefore, we recommend that future investigators be aware of this potential bias and consider using different sets of dosimeters for measuring noise levels during working and off-duty periods.

Although the weakness of the low number of subjects and potential differences between the groups of importance for the development of elevated blood pressure were not completely excluded, our findings generally supported the conclusion that there was a marginally transient effect and significantly sustained effect in normotensive adult autoworkers at occupational noise exposure above $70 \mathrm{dBA}$. For the transient effect, a 1-mm $\mathrm{Hg}$ increase in SBP was marginally associated with a 1-dBA increase in noise exposure. For the sustained effect, a $16-\mathrm{mm} \mathrm{Hg}$ increase in SBP was significantly associated with daily exposure to $85 \mathrm{dBA}$ TWA at work. Such findings are particularly important for an aging working population because blood pressure control was key to promoting adult workers' health. Accordingly, the current noise exposure guidelines of the American Conference of Governmental Industrial Hygienists $(\mathrm{ACGIH})^{28}$ and the International Organization for Standardization (ISO), ${ }^{29}$ which is based solely on hearing loss, might not be adequate for worker protection. We, therefore, recommend conducting further studies on blood pressure in relation to occupational noise and including the blood pressure effects in the future revision of noise exposure guidelines.

This was the first time that personal noise exposure was measured and the audiometric evaluation results were analyzed for the highexposure group in this company. We found the full-shift TWA noise exposure exceeded the 8-hour TWA of $85 \mathrm{dBA}$, which required employers to provide hearing protection devices (HPD) to workers in Taiwan. We also found that workers in the highexposure groups could have noiseinduced hearing loss besides noiseinduced blood pressure increases. After we found out these situations in the workplace, a hearing-conservation program has been recom- 
mended to the company immediately. We are still working closely with the company to evaluate this conservation program's effectiveness of protecting worker's health.

\section{References}

1. Thompson SJ. Epidemiology Feasibility Study: Effects of Noise on the Cardiovascular System. EPA Report No. 550/981-103A. Washington, DC: Environmental Protection Agency NTIS; 1981.

2. Thompson SJ. Effects of noise on the cardiovascular system: Appraisal of epidemiologic evidence. In: Rossi G, ed. Noise as a Public Health Problem: Proceedings of the Fourth International Congress. Milano: Centro Richerche E Studi Amplifon; vol 1, 1983:711-714.

3. Passchier-Vermeer W, Passchier WF. Noise exposure and public health. Environ Health Perspect. 2000;108(suppl 1): 123-131.

4. Jonsson A, Hansson L. Prolonged exposure to a stressful stimulus (noise) as a cause of raised blood pressure in man. Lancet. 1977;I:86-87.

5. Fouriad C, Jacquinet-Salord MC, Degoulet $\mathrm{P}$, et al. Influence of socioprofessional conditions on blood pressure levels and hypertension control. Epidemiologic study of 6665 subjects in the Paris district. Am J Epidemiol. 1984;120:72-86.

6. Verbeek JHAM, Van Dijk FJH, De Vries FF. Non-auditory effects of noise in industry. IV-A field study on industrial noise and blood pressure. Int Arch Occup Environ Health. 1987;59:51-54.

7. Wu TN, Ko YC, Chang PY. Study of noise exposure and high blood pressure in shipyard workers. Am J Ind Med. 1987; 12:431-438.

8. Zhao Y, Zhang S, Selvin S, et al. A dose-response relation for noise-induced hypertension. J Occup Environ Med. 1991;48:179-184.

9. Talbott EO, Gibson LB, Burks A, et al. Evidence for a dose-response relationship between occupational noise and blood pressure. Arch Environ Health. 1999;54: 71-78.

10. Tomei F, Fantini S, Tomao E, et al. Hypertension and chronic exposure to noise. Arch Environ Health. 2000;55: 319-325.

11. Andrén L, Hansson L, Björkman M, et al. Noise as a contributory factor in the development of elevated arterial pressure. Acta Med Scand. 1980;207:493498.

12. Holand S, Girard A, Laude D, et al. Effects of an auditory startle stimulus on blood pressure and heart rate in humans. J Hypertens. 1999;17:1893-1897.

13. Green MS, Schwartz K, Harari G, et al. Industrial noise exposure and ambulatory blood pressure and heart rate. J Occup Med. 1991;338:879-883.

14. Fogari R, Zoppi A, Corradi L, et al. Transient but not sustained blood pressure increments by occupational noise. An ambulatory blood pressure measurement study. J Hypertens. 2001;19:10211027.

15. Peterson EA, Augenstein JS, Tanis DC, et al. Noise raises blood pressure without impairing auditory sensitivity. Science. 1981;211:1450-1452.

16. Brinton TJ, Walls ED, Chio SS. Validation of pulse dynamic blood pressure measurement by auscultation. Blood Press Monit. 1998;3:121-124.

17. Brinton TJ, Walls ED, Yajnik AK, et al. Age-based differences between mercury sphygmomanometer and pulse dynamic blood pressure measurements. Blood Press Monit. 1998;3:125-129.

18. Littell RC, Milliken GA, Stroup WW, et al. Analysis of repeated measures data. In: SAS System for Mixed Models. Cary, NC: SAS Institute Inc; 1996:87-134.

19. Cnaan A, Laird NM, Slasor P. Tutorial in biostatistics - using the generalized linear model to analyze unbalanced repeated measures and longitudinal data. Stat Med. 1997;16:2349-2380.

20. Babisch W, Ising H, Gallacher JEJ, et al. Traffic noise and cardiovascular risk. The
Caerphilly study, first phase. Outdoor noise levels and risk factors. Arch Environ Health. 1988;436:407-414.

21. Babisch W, Ising H, Gallacher JEJ, et al. Traffic noise and cardiovascular risk. The Speedwell study, first phase. Outdoor noise levels and risk factors. Arch Environ Health. 1993;486:401-405.

22. Casto R, Nguyen T, Printz MP. Characterization of cardiovascular and behavioral responses to alerting stimuli in rats. Am J Physiol. 1989;256:R1121-R1126.

23. Baudrie V, Tulen JHM, Blanc J, et al. Autonomic components of the cardiovascular responses to an acoustic startle stimulus in rats. J Auton Pharmacol. 1997;17:303-309.

24. Melamed S, Bruhis S. The effects of chronic industrial noise exposure on urinary cortisol, fatigue, and irritability. $J$ Occup Environ Med. 1996;38:252-256.

25. Babisch W, Fromme H, Beyer A, et al. Increased catecholamine levels in urine in subjects exposed to road traffic noise. The role of stress hormones in noise research. Environ Int. 2001;26: 475-481.

26. Thompson SJ. Non-auditory health effects of noise: an updated review. In: Proceedings of Inter-Noise 96, August 1996, Liverpool, UK. In: Hill FA, Lawrence R, eds. St. Albans, UK: Institute of Acoustics; 1996:2177-2182.

27. Babisch W. Epidemiological studies of cardiovascular effects of occupational noise. A critical appraisal. Noise Health. 1998;1:24-39.

28. American Conference of Governmental Industrial Hygienists. Noise. In: Threshold Limit Values for Chemical Substances and Physical Agents and Biological Exposure Indices. Cincinnati: the Institute; 2002:104-106.

29. International Organization for Standardization. Acoustics: Determination of occupational noise exposure and Estimation of Noise-Induced Hearing Impairment. International Standard ISO 1999. Geneva: International Organization for Standardization; 1990. 